\title{
LEPIDÓPTEROS DE LA “QUEBRADA EL SAUCE”, CHIMBARONGO (COLCHAGUA), PARTE I: TORTRICIDAE
}

\author{
Francisco Urra ${ }^{1,2}$, David Gajardo ${ }^{3}$, Guillermo Valenzuela ${ }^{4}$, Roberto Vásquez ${ }^{5}$ \\ ${ }^{1}$ Área de Entomología, Museo Nacional de Historia Natural de Chile, Casilla 787, Santiago. francisco.urra@mnhn.gob.cl \\ ${ }^{2}$ PPG Biologia Animal, Departamento de Zoologia, Instituto de Biociências, Universidade Federal do Rio Grande do \\ Sul, Av. Bento Gonçalves 9500, Porto Alegre, RS, 91501-970, Brazil. \\ ${ }^{3}$ Facultad de Ciencias Veterinarias y Pecuarias, Universidad de Chile. \\ ${ }^{4}$ Programa de Magíster en Ciencias con Mención en Entomología, Universidad Metropolitana de Ciencias de la Educación. \\ ${ }^{5}$ Instituto de Ciencias de la Salud, Universidad de O'Higgins.
}

\section{RESUMEN}

Se entrega una lista de especies de la familia Tortricidae, recolectadas entre 2011 y 2019, en un remanente de bosque esclerófilo ubicado en Chimbarongo, provincia de Colchagua (Chile). Se documenta la presencia de 18 especies, pertenecientes a 12 géneros. Cuatro especies se registran por primera vez para esta zona.

Palabras clave: Bosque esclerófilo, lepidopterofauna, microlepidópteros, polillas, taxonomía.

\section{ABSTRACT}

Lepidoptera from "Quebrada EI Sauce”, Chimbarongo (Colchagua), Part I: Tortricidae. A list of species of the family Tortricidae, collected between 2011 and 2019, in a sclerophyllous forest remainder located in Chimbarongo, province of Colchagua (Chile), is provided. The presence of 18 species, belonging to 12 genera, is documented. Four species are registered for the first time for this area.

Key words: Lepidopterofauna, microlepidoptera, moths, sclerophyllous forest, taxonomy.

\section{INTRODUCCIÓN}

La zona central de Chile es un área de gran importancia biogeográfica por presentar una rica diversidad biológica y un elevado endemismo de especies (Armesto et al. 1996; Myers et al. 2000). Una de las principales amenazas a la biota de esta región, es la dramática modificación del ambiente por acción humana, situación que se ha agravado durante los últimos años con los frecuentes incendios forestales (González et al. 2020) y la prolongada sequía que afecta a la zona (Arroyo et al. 2019). A esto se suma la extrema vulnerabilidad de sus bosques nativos debido a la histórica sustitución del bosque original por especies de importancia económica (Hernández et al. 2006), relegando la sobrevivencia de la fauna a los remanentes de vegetación nativa (Bocaz et al. 2013). Debido a estas razones, se considera a la zona central de Chile como uno de los sitios prioritarios para la conservación de la biodiversidad (Myers et al. 2000). Considerando lo anterior, urge la necesidad de conocer la biodiversidad de estos ecosistemas; sólo el estudio y catalogación de los organismos presentes en estos ambientes permitirá dimensionar su valor y establecer las medidas destinadas para su conservación. 
Este estudio corresponde al primero de una serie de trabajos que tienen como objetivo conocer la fauna de lepidópteros presentes en un remanente de bosque esclerófilo ubicado en la "Quebrada El Sauce", localidad precordillerana de la comuna de Chimbarongo, situada en el límite sur de la provincia de Colchagua (Fig. 1). Esta zona es de especial interés taxonómico, pues corresponde a la localidad tipo de algunas especies de microlepidópteros descritas durante la última década, pertenecientes a las familias Oecophoridae, Depressariidae, Autostichidae, Tortricidae y Copromorphidae (Urra 2012, 2013, 2014a, 2014b, 2015, 2016, 2017a; Urra y Valenzuela 2020).

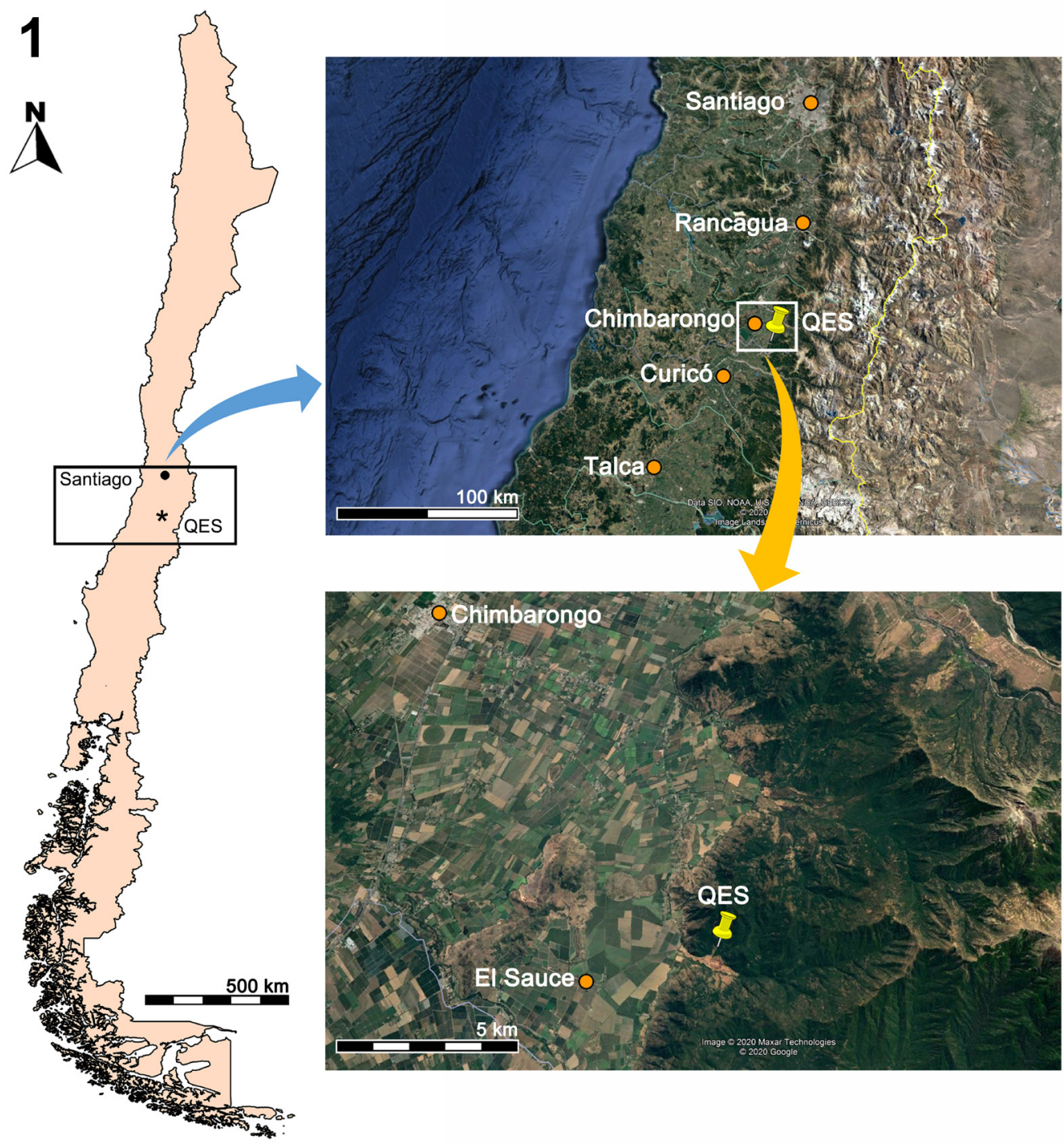

Figura 1. Ubicación de la localidad "Quebrada El Sauce" (QES) en Chile continental. El asterisco (*) y marca de posición corresponden a la georreferencia de uno de los puntos muestreados, 34²48'47"S 7056'2"W (GOOGLE EARTH PRO, 2020). 
En este trabajo, se documenta la presencia de especies de la familia Tortricidae, uno de los grupos de microlepidópteros más diverso en el mundo (Nieukerken et al. 2011). Esta familia reúne actualmente 11.365 especies descritas, agrupadas en 1.151 géneros (Gilligan et al. 2018) y en Chile está representada por 103 especies, de las cuales 97 corresponden a especies nativas, con un nivel de endemismo que alcanza el 87\% (Urra 2019, 2020); la tribu Cochylini es el grupo mejor representado de la subfamilia Tortricinae, incluyendo 83 especies conocidas, la mayor parte de ellas pertenecientes a la subtribu Euliina; en cuanto a su distribución geográfica, el $73 \%$ de las especies nativas se encuentra presente en la zona central del país (Brito y Vargas 2017; Cepeda 2017; Cepeda y González 2015; Razowski y Pelz 2010; Urra 2016, 2017b, 2020; Vargas 2011, 2019; Vargas et al. 2015; Vargas-Ortiz y Vargas 2018), asociadas principalmente a vegetación silvestre, aunque unas pocas especies actúan como plagas de cultivos frutales (González 2003).

\section{MATERIALES Y MÉTODOS}

Se examinó material entomológico recolectado en la “Quebrada El Sauce” (3448’47”S 7056’2”W), comuna de Chimbarongo, en la provincia de Colchagua, entre los años 2011 y 2019. Todos los ejemplares fueron capturados por el primer autor, en el lapso de tiempo indicado anteriormente y en distintos meses, usando trampa de luz blanca y negra UV, tipo sábana, alimentada con equipo electrógeno de $1.000 \mathrm{~W}$ de potencia; estos insectos fueron sacrificados con acetato de etilo y se montaron en extensores de alas usando minucias y alfileres entomológicos. Las estructuras genitales se estudiaron siguiendo la metodología propuesta por Lee y Brown (2006) y Robinson (1976), y se montaron en preparaciones permanentes con Euparal. Las fotografías se obtuvieron con cámara Sony Cybershot DSC-W830, bajo microscopio estereoscópico Olympus SZ51 y cámara Canon PowerShot SX30IS. El material examinado fue depositado en la Colección Nacional de Insectos del Museo Nacional de Historia Natural, Santiago, Chile.

Para cada especie registrada se entrega su distribución geográfica conocida en el país, indicada por las provincias o regiones administrativas y en paréntesis las localidades reportadas en la literatura; en el caso especies introducidas y de amplia distribución mundial, sólo se señala el rango de distribución geográfica reportado, según regiones administrativas. Bajo el subtítulo de "material examinado", se indica número de ejemplares según sexo y fechas de recolección; y se utilizan las siguientes abreviaturas transcritas desde las etiquetas: $\mathrm{PN}=$ parque nacional, $\mathrm{Q}=$ Quebrada, $\mathrm{RN}=$ reserva nacional y $\mathrm{SN}=$ santuario de la naturaleza. En las referencias no se incluyen las publicaciones referidas a descripciones de géneros, ni tampoco las de descripciones de aquellas especies introducidas.

\section{RESULTADOS}

Familia Tortricidae

Subfamilia Tortricinae

Accuminulia Brown, 1999

Accuminulia buscki Brown, 1999 (Fig. 2a)

Material examinado. CHILE COLCHAGUA, Chimbarongo Q El Sauce, 3448'47”S 7056'2'W, 7-XII2013 [1 ; ], 13-II-2016 [1 \%], Trampa luz col. F. Urra.

Distribución. Chile: Elqui (Coquimbo), Limarí (PN Bosque Fray Jorge), Choapa (Cuesta Cavilolén, El Naranjo, Nague), Petorca (25 km NNW Putaendo, $30 \mathrm{~km} \mathrm{~N} \mathrm{La} \mathrm{Ligua} 5 \mathrm{~km}$ S Las Palmas, Los Molles), Quillota (PN La Campana), Marga Marga (PN La Campana), Valparaíso (Valparaíso), Melipilla (La 
Viluma), Santiago (sin localidad precisa), Cordillera (Río Colorado), Cachapoal (Graneros), Cauquenes (RN Federico Albert, SN Reloca), Cauquenes (Alto Tregualemu) (Brown 1999; Cepeda 2014; Razowski y Pelz 2010). Se agrega la localidad de Quebrada El Sauce, en la provincia de Colchagua.

Comentarios. El género Accuminulia incluye dos especies conocidas, A. buscki y A. longiphallus Brown, 1999. Externamente, el macho de A. buscki se diferencia por carecer de escamas sexuales en la mitad basal

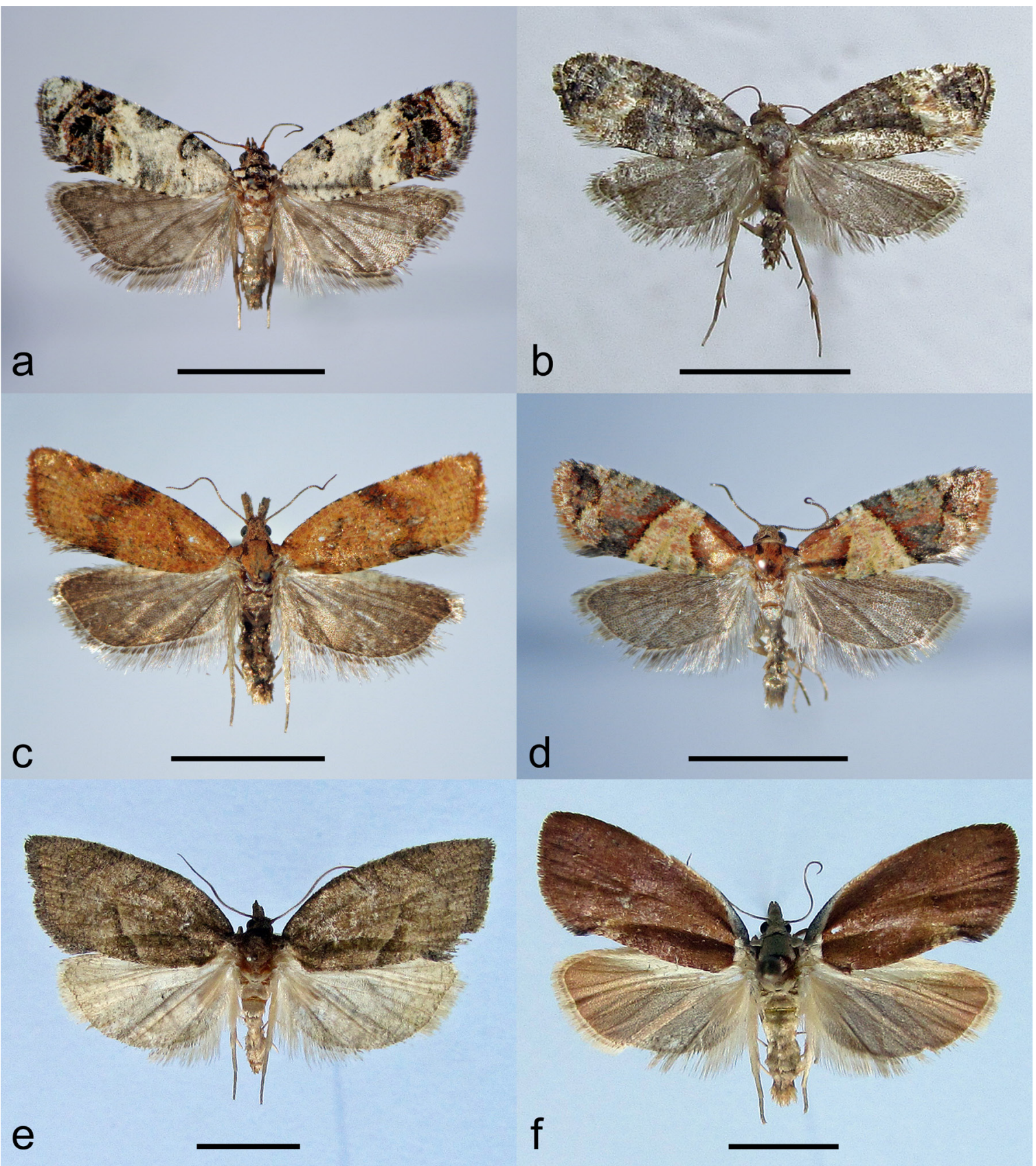

Figura 2. Tortricinae. a) Accuminulia buscki Brown, b) Acmanthina acmanthes (Meyrick), c) Eliachna digitana Brown \& McPherson, d) Haemateulia barrigana Razowski y González, e) Proeulia aethalea Obraztsov, f) Proeulia chrysopteris (Butler). 
del ala posterior, escamas que están presentes en A. longiphallus; respecto de las estructuras genitales, en $A$. buscki el aedeagus es corto y grueso, cerca de la mitad de la longitud de la valva, mientras que en $A$. longiphallus es curvo y de longitud cercana a la longitud de la valva; en las hembras, la presencia de una bolsa accesoria lateral en A. buscki permite distinguirla de A. longiphallus (Brown 1999).

Acmanthina Brown, 2000

Acmanthina acmanthes (Meyrick, 1931) (Fig. 2b)

Material examinado. CHILE COLCHAGUA, Chimbarongo Q. El Sauce, 3448'47'S 7056'2”W, 7-XII2013, Trampa luz col. F. Urra [1 $\left.\delta^{\top}\right]$.

Distribución. Chile: Los Andes (Río Blanco), Marga Marga (PN La Campana), Cordillera (Río Colorado), Cachapoal (RN Río Cipreses), Curicó (El Coigo, Potrero Grande, RN Radal Siete Tazas), Talca (RN Altos de Lircay), Linares (El Castillo Malcho), Cauquenes (Alto Tregualemu, Tregualemu), Diguillín (Las Trancas, Recinto), Biobío (8 km E Antuco, Caledonia, Laguna El Barco), Malleco (PN Nahuelbuta, Cordillera de las Raíces), Cautín (Pucón), Valdivia (Rincón de la Piedra), Llanquihue (Puerto Montt); Argentina (Brown 2000; Meyrick 1931; Razowski y Pelz 2010). Se agrega la localidad de Quebrada El Sauce, en la provincia de Colchagua.

Comentarios. El género Acmanthina incluye otras dos especies conocidas, A. albipuncta Brown, 2000 y A. molinana Razowski y Pelz, 2010. A. acmanthes puede distinguirse superficialmente de ambas por presentar un parche de escamas blancas subapical transverso (Brown 2000; Razowski y Pelz 2010).

\section{Eliachna Razowski, 1999}

Eliachna digitana Brown y McPherson, 2001 (Fig. 2c)

Material examinado. CHILE COLCHAGUA, Chimbarongo Q. El Sauce, 3448'47'S 7056'2”W 2-X-

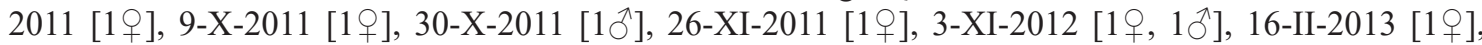
Trampa luz col. F. Urra.

Distribución. Chile: Santiago (Rinconada de Maipú), Cachapoal (Cajón de Lisboa, Pilay), Cauquenes (15 km S Curanipe, Alto Tregualemu), Itata (Carrizalillo, Iglesia de Piedra), Malleco (Río Manzanares), Cautín (Fundo El Coihue, Fundo Neltume), Valdivia (Rincón de la Piedra, Valdivia), Osorno (PN Puyehue), Llanquihue (Petrohué); Argentina (Brown y McPherson 2001; Razowski y Pelz 2010). Se agrega la localidad de Quebrada El Sauce, en la provincia de Colchagua.

Comentarios. El género Eliachna es similar al género Rebinea Razowski, 1986, debido a que las especies de ambos géneros presentan palpos labiales alargados y en los machos, la transtilla es esclerotizada con dos lóbulos dorsales y las valvas presentan una hendidura en el margen ventral. Eliachna se distingue de Rebinea por presentar en los machos, mayor desarrollo en el proceso del sacculus de la valva y por carecer de grandes cornuti en la vesica. El género Eliachna incluye otras dos especies, E. chileana Razowski, 1999 y E. hemicordata Brown y McPherson, 2002; E. digitana se distingue por presentar proceso del sacculus con menor desarrollo y que se curva ligeramente, comparado con las otras dos especies congéneres (Brown y McPherson 2001; Razowski y Pelz 2010).

Haemateulia Razowski, 1999

Haemateulia barrigana Razowski y González, 2003 (Fig. 2d)

Material examinado. CHILE COLCHAGUA, Chimbarongo Q. El Sauce, 3448’47’S 7056’2”W, 9-III2013 [1 ○] ], 21-II-2014 [2仓], Trampa luz col. F. Urra.

Distribución. Chile: Curicó (Los Niches, RN Radal Siete Tazas, Zapallar), Cauquenes (Alto Tregualemu), Diguillín (Las Trancas, Recinto), Biobío (8 km E Antuco), Malleco (PN Nahuelbuta), Cautín (Cerro Nielol, 27 km NE Villarrica); Argentina (Razowski y González 2003; Brown y Razowski 2003; Razowski y Pelz 
2010). Se agrega la localidad de Quebrada El Sauce, en la provincia de Colchagua, ampliando hacia el norte su distribución geográfica conocida.

Comentarios. El género Haemateulia incluye tres especies, H. barrigana, H. haematitis (Meyrick, 1931) y H. placens Razowski y Pelz, 2010. H. placens se distingue externamente de las otras dos especies por su coloración cremosa y manchas marrón difusas. H. barrigana se distingue de $H$. haematitis, por presentar valva más larga y uncus ligeramente espatulado (Brown y Razowski 2003; Razowski y Pelz 2010).

Proeulia Clarke, 1962

Proeulia aethalea Obraztsov, 1964 (Fig. 2e)

Material examinado. CHILE COLCHAGUA, Chimbarongo Q. El Sauce, 3448'47’S 7056’2'W, 22-XI-

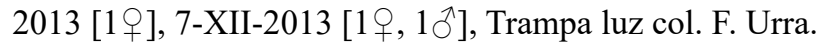

Distribución. Chile: Valparaíso (Valparaíso), Cordillera (La Obra) (Obraztsov 1964). Se agrega la provincia de Colchagua (Quebrada El Sauce), ampliando hacia el sur su distribución geográfica conocida.

Comentarios. Proeulia aethalea se distingue de otras especies del género por su coloración marrón oscuro en las alas anteriores; en el macho, la vesica presenta dos grupos de cornuti, el grupo superior con 11 unidades y el inferior con tres; mientras que en la hembra presenta lamella antevaginalis semicircular y cestum que nace desde un disco esclerotizado (Obraztsov 1964).

Proeulia chrysopteris (Butler, 1883) (Fig. 2f)

Material examinado. CHILE COLCHAGUA, Chimbarongo Q. El Sauce, 3448'51.4"S 7056'6.1"W, 18-II-2012, Trampa luz col. F. Urra [1 9 ]. CHILE COLCHAGUA, Chimbarongo Q. El Sauce, 3448'47'S

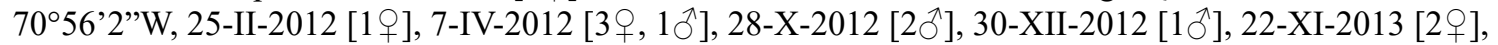

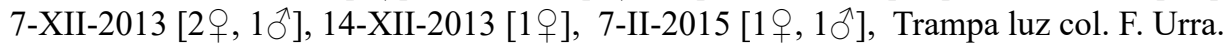

Distribución. Chile: Quillota (Quillota), Marga Marga (PN La Campana), Valparaíso (Valparaíso), Santiago (Santiago, RN Yerba Loca), Cordillera (Guayacán), Cachapoal (RN Río Cipreses, Termas de Cauquenes), Concepción (Concepción), Región de la Araucanía (sin localidad precisa), Valdivia (Valdivia), Llanquihue (Petrohué) (Butler 1883; Obraztsov 1964; Artigas 1994, Razowski y Pelz 2010). Se agrega la localidad de Quebrada El Sauce, en la provincia de Colchagua.

Comentarios. Proeulia chrysopteris presenta gran variación en su coloración, desde tonos anaranjados hasta el castaño oscuro; el patrón de coloración incluye una banda basal y una mancha subtriangular gris en el borde anal del ala anterior. En el macho, la valva presenta sacculus que se proyecta como un proceso agudo y la vesica tiene dos a cinco cornuti largos; en la hembra, la lamella antevaginalis tiene forma de "U" ancha (Obraztsov 1964; Razowski y Pelz 2010).

\section{Proeulia lentescens Razowski, 1995 (Fig. 3a)}

Material examinado. CHILE COLCHAGUA, Chimbarongo Q. El Sauce, 3448'47'S 7056'2”W, 23-XI2013 [1 $\bigcirc^{\wedge}$ ], 19-XI-2016 [1 + ], Trampa luz col. F. Urra.

Distribución. Chile: Coquimbo (Cuesta Cavilolén), Marga Marga (PN La Campana), Chacabuco (Altos de Chicauma), Santiago (Q de la Plata, SN Yerba Loca), Cordillera (San Alfonso), Diguillín (Las Trancas) (Cepeda y González 2015; Razowski 1995; Razowski y Pelz 2010). El material recolectado agrega la localidad de Quebrada El Sauce, en la provincia de Colchagua.

Comentarios. Externamente Proeulia lentescens es similar a P. sublentescens Razowski y Pelz, 2010, en cuanto al patrón de coloración de las alas anteriores. P. lentescens se distingue de $P$. sublentescens, porque en el macho, la vesica presenta dos cornuti de longitud similar, mientras que $P$. sublentescens presenta tres cornuti largos y uno más corto; en la hembra de $P$. lentescens la incisión del borde posterior 


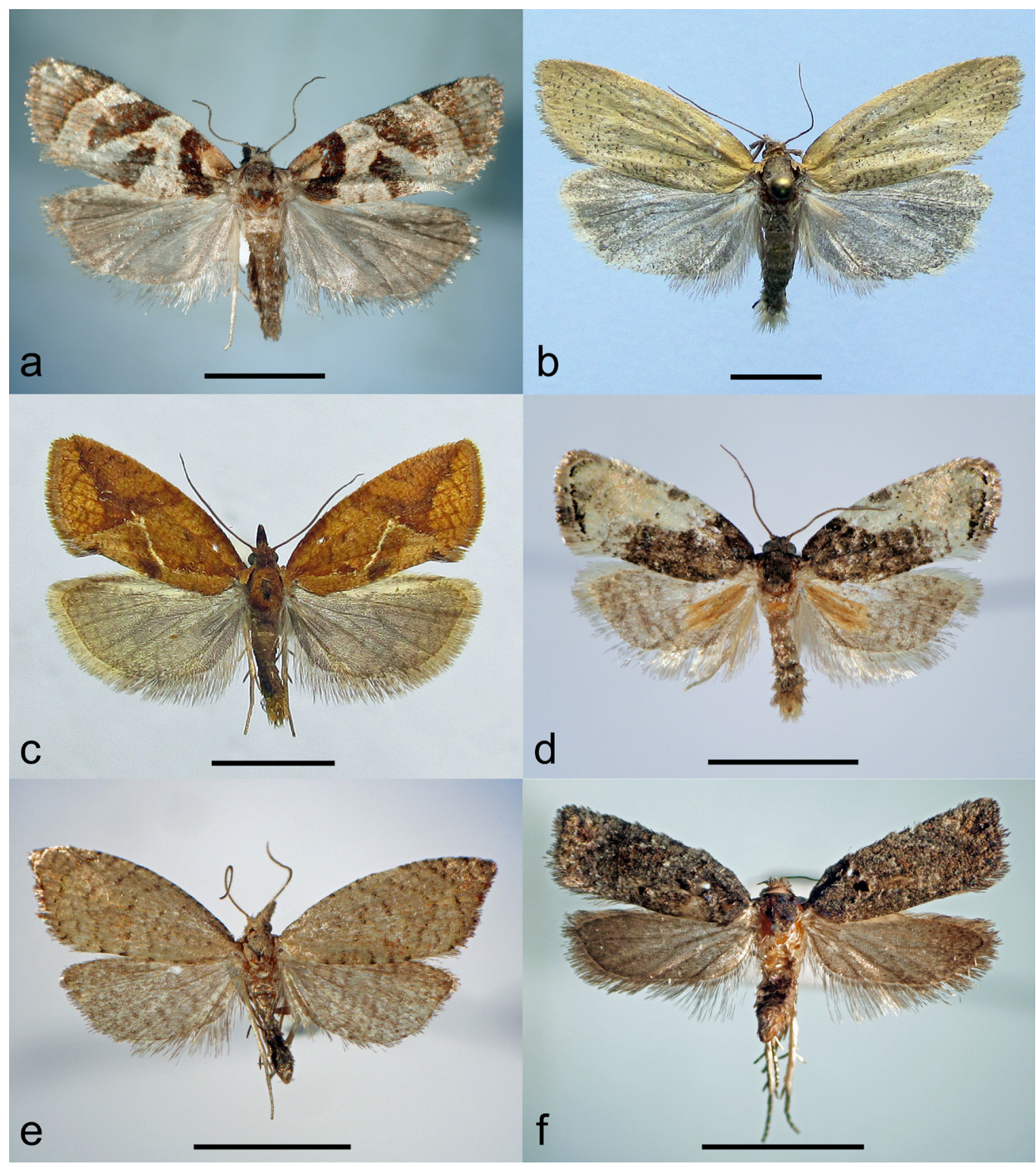

Figura 3. Tortricinae y Chlidanotinae. a) Proeulia lentescens Razowski, b) Proeulia leonina (Butler), c) Proeulia triquetra Obraztsov, d) Ptychocroca galenia Razowski, e) Rebinea erebina (Butler), f) Lypothora fernaldii (Butler)

de la lamella antevaginalis corresponde a un cuarto del largo de ésta, mientras que en $P$. sublentescens es de dos tercios (Razowski y Pelz 2010).

Proeulia leonina (Butler, 1883) (Fig. 3b)

Material examinado. CHILE COLCHAGUA, Chimbarongo Q. El Sauce, 3448’47’S 7056’’’W, 23-XI-2013 [1'], 7-XII-2013 [1 $\left.\delta^{\lambda}\right]$, Trampa luz col. F. Urra.

Distribución: Chile: Valparaíso (Valparaíso), Quillota (Quillota), Curicó (RN Radal Siete Tazas) (Butler 1883; Obraztsov 1964; Razowskiy Pelz 2010). El material recolectado agrega la localidad de Quebrada El Sauce, en la provincia de Colchagua. 
Comentarios. Externamente, Proeulia leonina es similar a P. gielisi Razowski y Pelz, 2010, pues ambas presentan coloración amarilla de las alas anteriores. No se conoce el macho de $P$. gielisi, pero la hembra de P. leonina se distingue por tener un saco lateral en el corpus bursae (Razowski y Pelz 2010).

Proeulia triquetra Obraztsov, 1964 (Fig. 3c)

Material examinado. CHILE COLCHAGUA, Chimbarongo Q. El Sauce, 3448'47'S 7056'2"W, 3-XII-

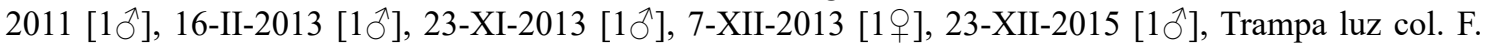
Urra.

Distribución. Chile: Región del Maule (sin localidad precisa), Diguillín (Chillán, San Ignacio), Concepción (Concepción), Región de la Araucanía (sin localidad precisa), Llanquihue (Lago Chapo) (Obraztsov 1964; Artigas 1994; Razowski 1995). Se agrega la localidad de Quebrada El Sauce, en la provincia de Colchagua, ampliando hacia el norte su distribución geográfica conocida.

Comentarios. Esta especie es fácilmente diferenciable de otras especies del género debido a su patrón de coloración; el ala anterior es castaño amarillento con tonos ferrugíneos e incluye una mancha subtriangular amarillenta en el borde anal. El macho presenta valva con sacculus reducido y tres cornuti largos y un grupo de 11 o 12 más pequeños en la vesica; la hembra presenta lamella antevaginalis con borde posterior sinuoso (Obraztsov 1964).

Ptychocroca Brown y Razowski, 2003

Ptychocroca galenia Razowski, 1999 (Fig. 3d)

Material examinado. CHILE COLCHAGUA, Chimbarongo Q. El Sauce, 3448’47’'S 7056'2”'W, 9-X-

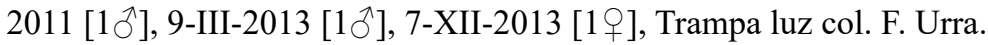

Distribución. Chile: Quillota (PN La Campana), Melipilla (La Viluma), Santiago (Maipú), Curicó (6 km E Molina, Potrero Grande, Río Teno, RN Radal Siete Tazas, Teno), Talca (RN Altos de Lircay), Linares (Embalse Bullileo, Malcho), Cauquenes (Alto Tregualemu, Pelluhue), Itata (Iglesia de Piedra), Diguillín (Las Trancas, Recinto, Shangrilá), Concepción (Concepción), Cautín (Fundo Chacamo) (Brown y Razowski 2003; Razowski 1999; Razowski y Pelz 2010). Se agrega la localidad de Quebrada El Sauce, en la provincia de Colchagua.

Comentarios. Externamente, las especies del género Ptychocroca son similares entre sí en cuanto a su patrón de coloración. Los machos de P. galenia presentan un penacho de escamas erectas cerca de la base de la costa del ala anterior, al igual que $P$. crocoptycha y $P$. simplex; sin embargo $P$. galenia puede distinguirse por la forma ligeramente atenuada de la valva hacia el ápice y la presencia de una espina en el borde ventral de ésta (Brown y Razowski 2003).

Rebinea Razowski, 1986

Rebinea erebina (Butler, 1883) (Fig. 3e)

Material examinado. CHILE COLCHAGUA, Chimbarongo Q. El Sauce, 3448'47’S 7056'2'W, 13-X-

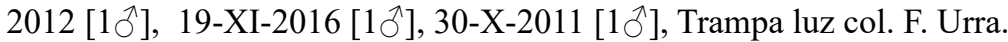

Distribución. Chile: Elqui (Coquimbo), Limarí (PN Bosque Fray Jorge), Choapa (Ñague), Los Andes (Curimón), Valparaíso (Valparaíso), Quillota (PN La Campana), Cordillera (Río Colorado), Cachapoal (Hacienda de Cauquenes, Pilay), Curicó (Río Teno), Cauquenes (Alto Tregualemu, Paso García), Diguillín (Las Trancas), Malleco (PN Tolhuaca), Valdivia (Rincón de la Piedra), Osorno (PN Puyehue), Llanquihue (Casa Pangue, Peulla, PN Vicente Pérez Rosales), Chiloé (Puntra); Argentina (Butler 1883; Brown y McPherson 2001; Razowski y Pelz 2010). Se agrega la localidad de Quebrada El Sauce, en la provincia de Colchagua. 
Comentarios. El género Rebinea incluye una segunda especie, $R$. brunnea Razowski y Pelz, 2010, de la que sólo se conocen hembras. La hembra de $R$. erebina presenta en el corpus bursae un área esclerotizada irregular con abundantes espículas, mientras que $R$. brunnea sólo presenta una simple esclerita lateral en el corpus bursae (Brown y McPherson 2001; Razowski y Pelz 2010).

\section{Subfamilia Chlidanotinae}

Lypothora Razowski, 1981

Lypothora fernaldii (Butler, 1883) (Fig. 3f)

Material examinado. CHILE COLCHAGUA, Chimbarongo Q. El Sauce, 3448'51.4”'S 7056'6.1”W, 18II-2012, Trampa luz col. F. Urra [1 $\left.\circ, 2^{`}\right]$. CHILE COLCHAGUA, Chimbarongo Q. El Sauce, 3448'47’S

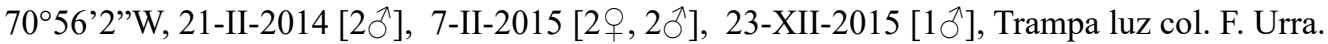

Distribución. Chile: Cauquenes (15 km S Curanipe), Cautín (Fundo El Coihue), Valdivia (Las Trancas W La Unión, Valdivia), Osorno (PN Puyehue) (Butler 1883; Brown y Adamski 2003; Razowski y Pelz 2010). Se agrega la localidad de Quebrada El Sauce, en la provincia de Colchagua, ampliando hacia el norte su distribución geográfica conocida.

Lypothora walsinghamii (Butler, 1883) (Fig. 4a)

Material examinado. CHILE COLCHAGUA, Chimbarongo Q. El Sauce, 3448’47’'S 7056'2”W, 23XII-2015 [1 §], 7-IV-2012 [1ठ], Trampa luz col. F. Urra.

Distribución. Chile: Petorca (Los Molles), Valparaíso (Valparaíso), Quillota (Cuesta El Melón), Cauquenes (17,5 km S Curanipe, Alto Tregualemu), Cautín (Cerro Nielol) (Butler 1883; Brown y Adamski 2003). Se agrega la localidad de Quebrada El Sauce, en la provincia de Colchagua.

Comentarios. El género Lypothora incluye sólo dos especies, L. walsinghamii y L. fernaldii, y es el único género de la subfamilia Chlidanotinae presente en Chile. El macho de L. walsinghamii presenta un cornutus grande y varios cornuti más pequeños en la vesica y la hembra tiene dos signa huecos y angostos con forma de cuerno y una banda esclerotizada en el extremo anterior del corpus bursae; por su parte el macho de $L$. fernaldii, presenta un cornutus único en la vesica, la hembra tiene dos signa anchos y huecos y su corpus bursae carece de banda esclerotizada (Brown y Adamski 2003).

\section{Subfamilia Olethreutinae}

Crocidosema Zeller, 1847

Crocidosema aporema Walsingham, 1914 (Fig. 4b)

Material examinado. CHILE COLCHAGUA, Chimbarongo Q. El Sauce, 3448'47’S 7056’2’W, 11-II-

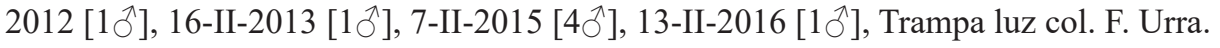

Distribución. Esta especie en Chile se distribuye desde la región de Arica y Parinacota hasta la región de Araucanía (Artigas 1994).

Crocidosema insulana Aurivillius, 1922 (Fig. 4c)

Material examinado. CHILE COLCHAGUA, Chimbarongo Q. El Sauce, 3448'47'S 7056'2”W, 19-

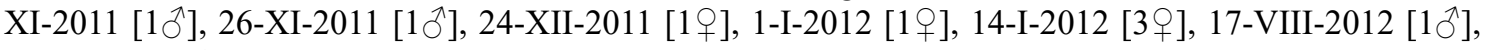
16-II-2013 [2ᄋ], Trampa luz col. F. Urra. 
Distribución. Chile: Elqui (21 km SE Coquimbo, 14 km S Vicuña), Limarí (15 km NW Combarbalá, 30 km NE Combarbalá, PN Bosque Fray Jorge), Choapa (8 km S Canela Baja), Petorca (8 km N Pedegua), Juan Fernández (Masatierra), Melipilla (8 km N Alhué), Curicó (RN Radal Siete Tazas), Linares (20 km E Linares valle del Río Ancoa), Biobío (28 km E Santa Bárbara, valle del río Huequecura) (Aurivillius 1922; Clarke 1965; Razowski y Pelz 2010). Se agrega la localidad de Quebrada El Sauce, en la provincia de Colchagua.

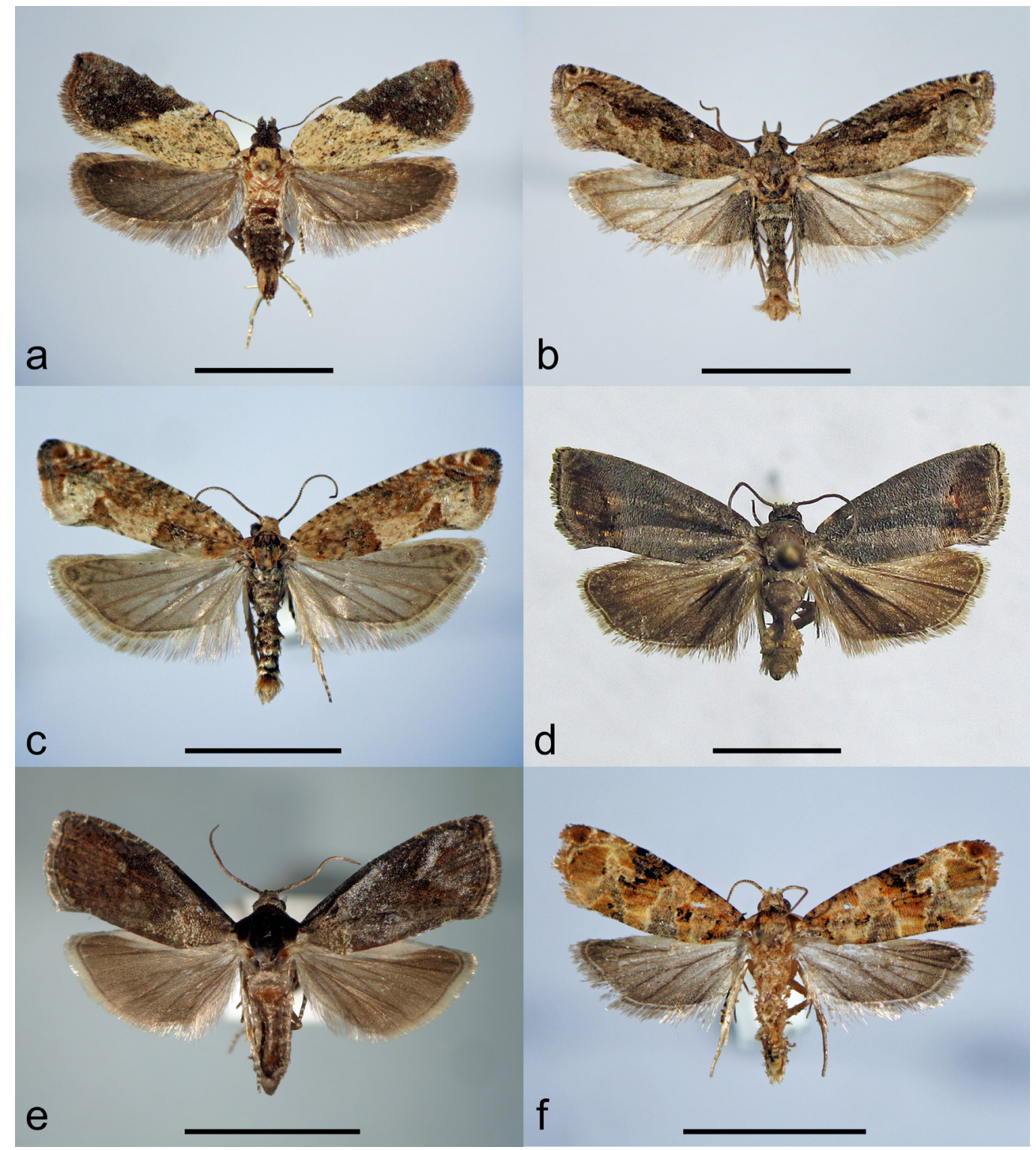

Figura 4. Chlidanotinae y Olethreutinae. a) Lypothora walsinghamii (Butler), b) Crocidosema aporema Walsingham, c) Crocidosema insulana Aurivillius, d) Cydia pomonella (Linnaeus), e) Grapholita molesta (Busck), f) Lobesia botrana (Denis y Schiffermüller). 
Comentarios. Externamente, el patrón de coloración del ala anterior de Crocidosema aporema y $C$. insulana es similar; sin embargo, en $C$. aporema los elementos son algo difusos y presenta manchas oscuras sobre la celda discal; en el ala posterior, C. aporema presenta numerosas escamas oscuras en la base de la región anal. Respecto de las estructuras genitales del macho, en C. aporema el cucullus de la valva se dirige hacia dorsal y presenta abundantes setas, mientras que en $C$. insulana, el cucullus de la valva se dirige hacia ventral y cuenta con espinas largas y gruesas en su ápice (Razowski y Pelz 2010). Recientemente, se describió una tercera especie para el género en Chile, Crocidosema nitsugai Vargas, 2019, proveniente de Putre (provincia de Parinacota) (Vargas 2019).

Cydia Hübner, 1825

Cydia pomonella (Linnaeus, 1758) (Fig. 4d)

Material examinado. CHILE COLCHAGUA, Chimbarongo Q. El Sauce, 3448'47'S 7056'2'W, 14-I2012, Trampa luz col. F. Urra $\left[1\right.$,, $\left.1{ }^{\Uparrow}\right]$.

Distribución. Esta especie es cosmopolita; en Chile se distribuye desde la Región de Arica y Parinacota a la Región de Los Lagos (Artigas 1994).

Comentarios. Esta especie es fácilmente reconocible por su coloración gris con una gran mancha cobriza en el extremo del ala anterior. Su recolección en este ambiente se debería a la presencia de algunos de sus hospederos como los ciruelos asilvestrados (Prunus sp.) (González 2003).

Grapholita Treitschke, 1829

Grapholita molesta (Busck, 1916) (Fig. 4e)

Material examinado. CHILE COLCHAGUA, Chimbarongo Q. El Sauce, 3448’47’S 7056'2'W, 14-I2012, Trampa luz col. F. Urra [1 $\left.\oint^{\Uparrow}\right]$.

Distribución. Esta especie es de origen oriental; en Chile se distribuye desde la Región de Antofagasta a la Región de Los Lagos (Artigas 1994).

Comentarios. Esta especie se diferencia fácilmente de Cydia pomonella por su menor tamaño y por su coloración gris oscuro y las estrías de color blanco en la costa del ala anterior. Su recolección en este ambiente obedecería a la presencia de algunos de sus hospederos como los ciruelos asilvestrados (Prunus sp.) (González, 2003).

Lobesia Guenée, 1845

Lobesia botrana (Denis y Schiffermüller, 1775) (Fig. 4f)

Material examinado. CHILE COLCHAGUA, Chimbarongo Q. El Sauce, 3448’47’S 7056’2”W, 13-II2016, Trampa luz col. F. Urra [1 + ].

Distribución. Esta especie en Chile se distribuye desde la región de Atacama hasta la región de Los Lagos (Álvarez et al. 2016).

Comentarios. Lobesia botrana es una especie polífaga y plaga clave para la vid en Chile. La recolección de este lepidóptero en ambientes naturales se debería a la presencia de algunos de sus hospederos secundarios, como los ciruelos asilvestrados (Prunus sp.) (Álvarez et al. 2016; Coscollá 1997). Externamente, puede ser confundida con especies de pequeño tamaño como Accuminulia buscki y Haemateulia barrigana, sobre todo si los ejemplares han perdido parte de sus escamas; sin embargo, el examen de las estructuras genitales permite separarlas rápidamente. 


\section{DISCUSIÓN}

Este estudio arrojó la presencia de 18 especies de la familia Tortricidae, 15 de éstas corresponden a especies nativas que se distribuyen en la zona central y sur del país; además, para cuatro de estas especies, Haemateulia barrigana, Proeulia aethalea, Proeulia triquetra y Lypothora fernaldii, se amplía el rango de distribución geográfica conocida. Respecto de las especies exóticas, Cydia pomonella, Grapholita molesta y Lobesia botrana, éstas corresponden a plagas de cultivos frutales de importancia primaria; su presencia en el lugar se explica por la existencia de hospederos exóticos asilvestrados, en este caso ciruelos (Prunus sp.).

Anteriores trabajos en la zona han permitido describir nuevos géneros y especies de microlepidópteros, entre ellos un tortrícido (Parvulia luteocastanea Urra, 2016), lo que da a entender que la lepidopterofauna de estos ambientes ha sido escasamente estudiada, y que eventualmente, nuevas entidades taxonómicas podrían ser descubiertas.

Es probable que futuras prospecciones en el lugar permitan agregar nuevos registros de tortrícidos y de lepidópteros de otras familias, que serán publicados en posteriores trabajos de esta serie, y así avanzar en la preparación de un inventario de especies para la zona. La presencia de un remanente de bosque esclerófilo poco intervenido, hace a la Quebrada El Sauce una localidad interesante de estudiar desde el punto de vista faunístico, permitiéndonos conocer y valorar la biota del ecosistema mediterráneo de Chile central.

\section{AGRADECIMIENTOS}

Se agradece a la Sra. Pilar Vial por facilitar el acceso al predio donde se ubica la "Quebrada El Sauce"; y a los señores Aldo Morán, Ángel Sánchez y Manuel Urra, por su colaboración en las actividades de recolección.

\section{REFERENCIAS BIBLIOGRÁFICAS}

ÁlVAREZ, A. A., M. P. AZÓCAR, C. ROZAS, Y. NABALON, K. FLORIO y A. GARRIDO. 2016. Lobesia botrana en Chile. Resultados del Programa Nacional de Lobesia botrana del Servicio Agrícola y Ganadero, Temporada 20142015. Simiente, 86(3-4): 33-44.

ARMESTO, J., P. LEÓN-LOBOS y M. K. ARROYO. 1996. Los bosques templados del sur de Chile y Argentina: Una isla biogeográfica. In ARMESTO, J., C. VILLAGRÁN y M. K. ARROYO (Eds.). Ecología de los bosques nativos de Chile. Editorial Universitaria. Santiago. 469 p.

ARROYO, M. T. K., A. PAUCHARD, D. ALARCÓN, J. ARMESTO, F. BOZINOVIC, R. BUSTAMANTE, C. ECHEVERRÍA, S. A. ESTAY, R. A. GARCÍA, A. GAXIOLA, M. MIRANDA, P. PLISCOFF, D. ROZAS, C. SALAS-ELJATIB y R. ROZZI. 2019. Capítulo 1, Impactos del cambio climático en la biodiversidad y las funciones ecosistémicas en Chile. En Marquet, P. A. et al. (Eds.), Biodiversidad y cambio climático en Chile: Evidencia científica para la toma de decisiones. Informe de la mesa de Biodiversidad. Santiago: Comité Científico COP25; Ministerio de Ciencia, Tecnología, Conocimiento e Innovación. 318 p.

ARTIGAS, J. N. 1994. Entomología Económica. Ediciones Universidad de Concepción; Concepción, Chile, Vol. II. $943 \mathrm{p}$.

AURIVILLIUS, C. 1922. Tortricidae p. 301. In: Skottsberg, C. (ed.). The Natural History of Juan Fernandez and Eastern Island 3(2): 256-269.

BOCAZ-TORRES, P., A. O. ANGULO y L. E. PARRA. 2013. Diversidad de macrolepidópteros nocturnos de la Reserva Nacional Nonguén (Región del Biobío, Chile) (Insecta: Lepidoptera). SHILAP Revista de Lepidopterología, 41 (163): 337-347.

BRITO, R. y H. A. VARGAS. 2017. A new leaf-tying Episimus Walsingham (Lepidoptera: Tortricidae) feeding on the vulnerable tree Haplorhus peruviana (Anacardiaceae) in the Atacama Desert of northern Chile. Studies on Neotropical Fauna and Environment, DOI: 10.1080/01650521.2017.1413824.

BROWN, J. W. 1999. A new genus of Tortricid moths (Tortricidae: Euliini) injurious to grapes and stone fruits in Chile. Journal of the Lepidopterists' Society 53(2): 60-64. 
BROWN, J. W. 2000. Acmanthina: A new genus of tortricid moths (Lepidoptera: Tortricidae: Euliini) from Chile and Argentina. Journal of the New York Entomological Society, 108:106-113.

BROWN, J. W. y ADAMSKI, D. 2003. A taxonomic review of Lypothora Razowski (Lepidoptera: Tortricidae: Polyorthini) with comments on polymorphism and a new synonymy. Pan.Pacific Entomologist 79(2): 128-134.

BROWN, J. W. y T. Y. MCPHERSON. 2001. Review of Rebinea Razowski and Eliachna Razowski (Tortricidae: Euliini) - sister groups endemic to Chile and Argentina. Journal of Lepidopterist' Society 55(4): 129-139.

BROWN, J. W. y J. RAZOWSKI. 2003. Description of Ptychocroca, a new genus from Chile and Argentina, with comments on the Bonagota Razowski group of genera (Lepidoptera: Tortricidae: Euliini). Zootaxa 303: 1-31.

BUTLER, A. G. 1883. Heterocerous Lepidoptera collected in Chili by Thomas Edmonds, Esq. Part IV Pyrales and Micros. Transactions of the Entomological Society of London 1883(1): 49-90.

CEPEDA, D. E. 2014. Descripción del último estado larvario de Accuminulia buscki, especie de Tortricidae (Lepidoptera: Euliini) de importancia económica en Chile. Revista Chilena de Entomología 39: 23-27.

CEPEDA, D. E. 2017. Una nueva especie del género Eugnosta Hübner, de Chile (Lepidoptera: Tortricidae). Insecta Mundi, 0565: 1-6.

CEPEDA, D. E. y R. H. GONZÁLEZ. 2015. Nueva especie del género Proeulia Clarke, con registros adicionales de distribución geográfica para cinco especies (Lepidoptera: Tortricidae). Revista Chilena de Entomología, 40: 1-8.

CLARKE, J. F. G. 1965. Microlepidoptera of the Juan Fernandez Islands. Proceedings of the United States National Museum 117(3508): 1-105.

COSCOLLÁ, R. 1997. La polilla del racimo dela vid (Lobesia botrana Den. Y Schiff.). Generalitat Valenciana, Conselleria de Agricultura, Pesca y Alimentación, Valencia, España. 613 p.

GILLIGAN, T. M., J. BAIXERAS y J. W. BROWN. 2018. T@RTS: Online World Catalogue of the Tortricidae (Ver. 4.0). http://www.tortricid.net/catalogue.asp. Consultado 5 de agosto de 2020.

GONZÁlez, M. E., R. SAPIAINS, S. GÓMEZ-GONZÁlEZ, R. GARREAUD, A. MIRANDA, M. GAlleguillos, M. JACQUES, A. PAUCHARD, J. HOYOS, L. CORDERO, F. VÁSQUEZ, A. LARA, P. ALDUNCE, V. DELGADO, R. ARRIAGADA, A. M. UGARTE, A. SEPÚLVEDA, L. FARÍAS, R. GARCÍA, R. RONDANELLI, R. PONCE, F. VARGAS, M. ROJAS, J. P. BOISIER, J. CARRASCO, C. LITTLE, M. OSSES, C. ZAMORANO, I. DÍAZ-HORMAZÁBAL, A. CEBALLOS, E. GUERRA, M. MONCADA y I. CASTILLO. 2020. Incendios forestales en Chile: causas, impactos y resiliencia. Centro de Ciencia del Clima y la Resiliencia (CR)2, Universidad de Chile, Universidad de Concepción y Universidad Austral de Chile. 84 p.

GONZÁLEZ, R. 2003. Las polillas de la fruta en Chile (Lepidoptera: Tortricidae; Pyralidae). Universidad de Chile, Serie Ciencias Agronómicas N 9, Santiago, Chile. 188 p.

GOOGLE EARTH PRO 7.3.4.8248. 2020. Chimbarongo, Chile. 3448’47’S 7056’2”W. Alt. ojo 25 km. Límites. NOAA, U.S. Navy, NGA, GEBCO. Maxar Technologies 2020. http:/www.google.com/earth/ [Agosto, 2020]

HERNÁNDEZ, J., C. L. DE LA MAZA y C. ESTADES. 2006. Biodiversidad: Manejo y Conservación de Recursos Forestales. Editorial Universitaria, Santiago. 803 p.

LEE, S. M. y R. L. BROWN. 2006. A new method for preparing slide mounts of whole bodies of microlepidoptera. Journal of Asia-Pacific Entomology, 9(3): 249-253.

MEYRICK, E. 1931. Microlepidoptera from South Chile and Argentina. Anales Museo Nacional de Historia Natural, Buenos Aires 36: 377-415.

MYERS, N., R. MITTERMEIER, C. MITTERMEIER, G. DA FONSECA y J. KENT, J. 2000. Biodiversity hotspots for conservation priorities. Nature, 403: 853-858.

NIEUKERKEN, E.J. VAN, L. KAILA, I. J. KITCHING, N. P. KRISTENSEN, D. C. LEES, J. MINET, C. MITTER, M. MUTANEN, J. C. REGIER, T. J. SIMONSEN, N. WAHLBERG, S. H. YEN, R. ZAHIRI, D. ADAMSKI, J. BAIXERAS, D. BARTSCH, B. A. BENGTSSON, J. W. BROWN, S. R. BUCHELI, D. R. DAVIS, J. DE PRINS, W. DE PRINS, M. E. EPSTEIN, P. GENTILI-POOLE, C. GIELIS, P. HATTENSCHWILER, A. HAUSMANN, J. D. HOLLOWAY, A. KALLIES, O. KARSHOLT, A. Y. KAWAHARA, S. KOSTER, M. V. KOZLOV, J. D. LAFONTAINE, G. LAMAS, J. F. LANDRY, S. M. LEE, M. NUSS, K. T. PARK, C. PENZ, J. ROTA, A. SCHINTLMEISTER, B. C. SCHMIDT, J. C. SOHN, M. A. SOLIS, G. M. TARMANN, A. D. WARREN, S. WELLER, R. V. YAKOVLEV, V. V. ZOLOTUHIN y A. ZWICK. 2011 Order Lepidoptera Linnaeus, 1758, pp. 212-221. In: ZHANG, Z.-Q. (Ed.), Animal biodiversity: An outline of higher-level classification and survey of taxonomic richness. Zootaxa, 3148: 1-237. 
OBRAZTSOV, N. S. 1964. Neotropical Microlepidoptera V, Synopsis of the species of the genus Proeulia from central Chile (Lepidoptera: Tortricidae). Proceedings of the United States National Museum, 116(3501), 183-195.

RAZOWSKI, J. 1995. Proeulia Clarke, 1962, the Western Neotropical Tortricidae genus (Lepidoptera), with descriptions of five new species and two allied genera. Acta Zoologica Cracovensia, 38(2): 271-293.

RAZOWSKI, J. 1999. Euliini (Lepidoptera: Tortricidae) of Chile. Polskie Pismo Entomologiczne, 68: 69-90.

RAZOWSKI, J. y R. H. GONZÁLEZ. 2003. Descriptions of two Chilean Euliini species (Lepidoptera: Tortricidae). SHILAP Revista de Lepidopterología, 31(121): 61-64.

RAZOWSKI, J. y V. PELZ. 2010. Tortricidae from Chile (Lepidoptera: Tortricidae) SHILAP Revista de Lepidopterología, 38(149): 5-55.

ROBINSON, G. S. 1976. The preparation of slides of Lepidoptera genitalia with special reference to microlepidoptera. Entomologist's Gazette, 27: 127-132.

URRA, F. 2012. Dos nuevas especies del género Dita (Lepidoptera: Oecophoridae). Revista Chilena de Entomología, 37: 67-73.

URRA, F. 2013. Contribución al conocimiento de los Oecophoridae (Lepidoptera: Gelechioidea) de Chile central. Acta Entomológica Chilena, 33(1-2): 31-46.

URRA, F. 2014a. Aidabella, nuevo género de Oecophoridae (Lepidoptera: Gelechioidea) de Chile central. Boletín del Museo Nacional de Historia Natural, Chile, 63: 33-42.

URRA, F. 2014b. Un nuevo género chileno de Depressariidae (Lepidoptera: Gelechioidea). Boletín del Museo Nacional de Historia Natural, Chile, 63: 101-110.

URRA, F. 2015. Revisión de los géneros Hyperskeles Butler y Eraina Clarke (Lepidoptera: Autostichidae). Boletín del Museo Nacional de Historia Natural, Chile, 64: 25-40.

URRA, F. 2016. Parvulia, nuevo género de Tortricidae (Lepidoptera) de la zona central de Chile. Boletín del Museo Nacional de Historia Natural, Chile, 65: 161-167.

URRA, F. 2017a. Corita attenboroughi sp. nov., nueva especie de Oecophoridae (Lepidoptera: Gelechioidea) de Chile central. Biodiversity and Natural History, 3(1): 29-33.

URRA, F. 2017b. Foikeulia razowskii, nuevo género y nueva especie de tortrícido (Lepidoptera: Tortricidae) asociada a Drimys winteri (Winteraceae). Revista Chilena de Entomología, 43: 47-54.

URRA, F. 2019. Estado actual del conocimiento de la diversidad taxonómica de los microlepidópteros chilenos. En: Libro de Resúmenes XL Congreso Nacional de Entomología, 2019, Concepción, Chile. pp 48.

URRA, F. 2020. Natria mauritius, nuevo género y nueva especie de tortrícido (Lepidoptera: Tortricidae) de la cordillera de Nahuelbuta, Chile. Revista Chilena de Entomología, 46(3): 357-364.

URRA, F. y G. VALENZUELA. 2020. Contribución al conocimiento de la familia Copromorphidae (Lepidoptera: Carposinoidea) en Chile. Boletín del Museo Nacional de Historia Natural, Chile, 69(1): 33-47.

VARGAS, H. A. 2011. A new species of Eccopsis Zeller (Lepidoptera, Tortricidae) from the coastal valleys of northern Chile, with the first continental record of E. galapagana Razowski \& Landry. Revista Brasileira de Entomologia, 55(2): 216-218.

VARGAS, H. A. 2019. A new species of Crocidosema Zeller (Lepidoptera, Tortricidae) from the Andes of northern Chile. Nota Lepidopterologica, 42(2): 129-136. DOI 10.3897/nl.42.38341

VARGAS, H. A., P. POLLO, D. S. BASILIO, G. L. GONÇANVES y G. R. P. MOREIRA. 2015. A new cecidogenous species of Eugnosta Hübner (Lepidoptera: Tortricidae) associated with Baccharis salicifolia (Asteraceae) in the northern Chilean Atacama Desert: Life-history description and phylogenetic inferences. Zootaxa, 3920(2): 265-280.

VARGAS-ORTIZ, M. y H. A. VARGAS. 2018. A new species of Strepsicrates Meyrick (Lepidoptera: Tortricidae) from the Atacama Desert of northern Chile previously misidentified as S. smithiana Walsingham. Zootaxa, 4370(5): 569-579. 